\title{
Processo de trabalho do sistema de referência e contra-referência da área da Odontologia
}

\author{
Flávio Renato Reis de Moura*, Paulo Renato Christoff Bissacotti**, Francisco Avelar \\ Bastos*, Rubem Beraldo dos Santos*, Flávio Fernando Demarco***, Maximiano \\ Ferreira Tovo****
}

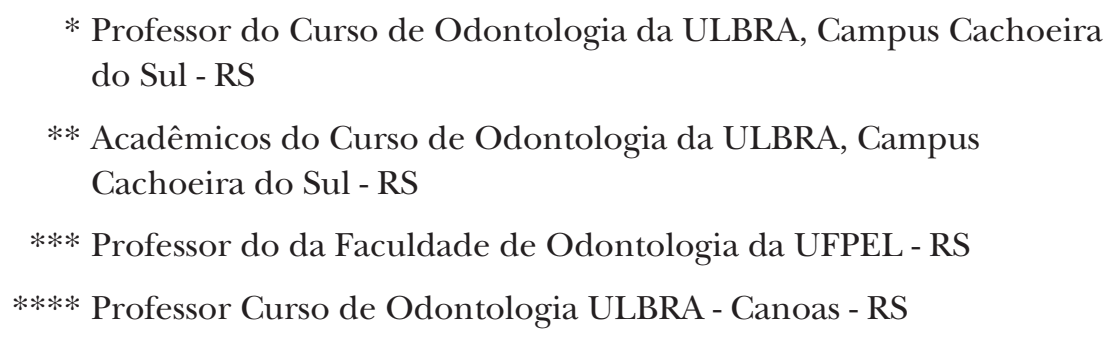

\section{RESUMO}

O objetivo do presente estudo foi analisar o processo de trabalho do sistema de referência e contrareferência da área da Odontologia. Para subsidiar a análise normativa e comparativa foram realizadas entrevistas compostas de oito perguntas fechadas dirigidas aos Secretários Municipais de Saúde (SMS) e Coordenadores das Unidades Básicas de Saúde (UBS) de uma Coordenadoria Regional da Saúde (CRS) do RS composta por doze municípios. Após a realização das entrevistas os dados foram tabulados em freqüências simples. Para análise comparativa as respostas obtidas nas SMS(s) foram consideradas como padrão ouro para emitir juízo de valor. No final da análise foram identificadas potencialidades e limitações do processo de trabalho da interface de governabilidade municipal. Conclui-se que o processo de trabalho do sistema de referência e contra-referência da área da odontologia deve ser planejado pelos gestores das SMS(s) e UBS(s) com a coordenação da CRS para adequar as necessidades de cada município.

\section{DECRITORES}

Referência. Atenção secundária. Saúde bucal.

$\mathbf{N}$ a evolução histórica do Sistema Nacional de Saúde do Brasil a Saúde Bucal vem buscando ocupar espaço no cenário da saúde com vistas ao conceito ampliado de saúde. Atualmente com um melhor entendimento dos princípios do Sistema Único de Saúde (SUS) o Ministério da Saúde publicou em 2001 a
Portaria $267^{1}$ que possibilitou a inserção a Equipe de Saúde Bucal na Estratégia de Saúde da Família como premissa de ampliar o acesso da população aos serviços públicos de saúde. Na progressão de uma "linha do tempo" e no trabalho da Equipe Técnica do Ministério da Saúde, em 2004 foram publicadas as Diretrizes da Política Nacional de Saúde Bucal ${ }^{2}$ visando à ampliação do acesso da população ao atendimento odontológico em nível da atenção básica e de média complexidade.

Considerando o processo de construção da Política Nacional de Saúde Bucal em julho de 2004, através da Portaria Ministerial $1572^{3}$ foi normatizado o financiamento para implantação dos Centros de Especialidades Odontológicas (CEOs) para contemplar o princípio da integralidade da atenção à Saúde Bucal. A referida Portaria estabelece os tipos de CEO(s) que podem ser implantados e suas respectivas cifras de financiamento para implantação e custeio. A legislação prevê como responsabilidades dos CEOS(s) de realizar procedimentos nas áreas de periodontia, cirurgia, endodontia e atendimento de pacientes portadores de necessidades especiais sendo classificadas como Clínicas Especializadas quando cadastradas no Sistema de Cadastro Nacional de Estabelecimento de Saúde (CNES).$^{5}$ No período de 4 anos, ou seja, até 2008 foram implantados 674 Centros de Especialidades Odontológicas beneficiando mais de 575 municípios em todo o Brasil $^{6}$ atingindo em 2009 o número de 808 unidades em funcionamento. ${ }^{7} \mathrm{Na}$ seqüência, para o bom funcionamento das unidades de referên- 
cia a interface entre o atendimento da atenção primária e secundária deve ser coordenada/estruturada para que não haja descontinuidade no serviço proporcionando efetividade e eficiência no desempenho das ações da atenção secundária. ${ }^{8}$

A nova política para saúde bucal traz consigo a oportunidade da população SUS-dependente evitar as perdas dentárias e executar a reabilitação quando necessário. Assim contextualizado, os CEOs e os Laboratórios Regionais de Prótese Dentária (LRPD) caracterizam a ampliação e qualificação dos centros de referência e contra-referência para as Equipes de Saúde Bucal da Estratégia de Saúde da Família bem como para as Unidades Básicas de Saúde que prestam atendimento odontológico. ${ }^{6}$

As Portarias $267,{ }^{1} 1.571^{4}$ e $1572^{3}$ oportunizam aos gestores estaduais e municipais na revitalização da saúde bucal dos usuários do SUS, pautados em um novo modelo assistencial à saúde com normas e legislação específica. Para potencializar estes documentos normatizados pelo Ministério da Saúde há necessidade que os gestores tenham qualificação necessária para utilizar as ferramentas (Portarias) e ao mesmo tempo devem ter conhecimento da situação problema do sistema de referência na Saúde Bucal de suas comunidades partícipes de seu pleito eleitoral. ${ }^{59}$ Portanto, é importante analisar o sistema de referência e contra-referência da área da odontologia nas Coordenadorias Regionais de Saúde para fomentar os gestores na reflexão da eficiência e/ou a inexistência deste sistema no município/região dando continuidade a ampliação e valorização da evidente Política Nacional de Saúde Bucal. ${ }^{8}$ Para o bom andamento da implementação da atual política é fundamental considerar que a taxa de pacientes que necessitam do sistema de referência e contra-referência da odontologia podem estar associados a fatores como:

- nível de necessidades dos pacientes,

- idade e a presença de cirurgiões-dentistas com pós-graduação atuante na rede básica de serviços odontológicos,

- tipo de remuneração e habilidades dos profissionais bem como a distância das unidades básicas daquela que presta o atendimento de média complexidade. ${ }^{10}$

Considerando que poucos trabalhos têm avaliado o processo de construção da atenção secundária ${ }^{6} \mathrm{e}$ que o gestor como o ator social que planeja e organiza o serviço a ser prestado à população, possibilitando impactar positivamente no quadro epidemiológico de saúde bucal, o presente estudo tem como objetivo analisar o processo de trabalho do sistema de referência e contra-referência da área da Odontologia de uma Coordenadoria Regional da Saúde do RS.

\section{METODOLOGIA}

O estudo foi do tipo descritivo, quantitativo e normativo. ${ }^{5}$ Uma Coordenadoria Regional da Saúde do Estado do Rio Grande do Sul (CRS-RS). Os doze (12) municípios pertencentes à área de abrangência da Coordenadoria foram incluídos no estudo. A coleta de dados foi realizada através de entrevistas agendadas previamente por um único pesquisador treinado que utilizou dois questionários pré-testados em dois momentos distintos com intervalo de 7 dias. Os questionários foram pré-testados com dois professores da área da saúde bucal coletiva.

Após o pré-teste e as devidas retificações um questionário foi utilizado para entrevistar o gestor responsável pela Secretaria Municipal da Saúde (SMS) e outro para a pessoa que Coordenava a Unidade Básica de Saúde (UBS) indicada pela SMS de cada município. Com o intuito de analisar comparativamente a existência do processo de trabalho na CRS-RS cinco perguntas foram idênticas direcionadas à SMS e UBS abordando as seguintes variáveis:

- existência do sistema de referência e contra-referência em Odontologia no município,

- qual (ais) unidade (es) de referência do município,

- quais são especialidades odontológicas contempladas nos locais de referência,

- quais são os critérios utilizados para realizar os encaminhamentos e

- se existe um sistema de acompanhamento pela UBS dos tratamentos realizados pelo local de referência.

Para completar a análise do processo de trabalho nas UBS(s) foi questionado aos coordenadores (as) sobre qual (is) era (am) o (os) local (is) que utilizavam como referência para demanda qualitativa de procedimentos odontológicos diagnosticados pelo cirurgião-dentista. Ainda para identificar o processo de trabalho coordenado pelos SMS foi questionado a este nível de gestão:

- qual era o destino dos pacientes após o tratamento realizado no centro de referência e

- qual era o protocolo seguido para volta dos pacientes à UBS(s). 
Naquelas questões em que as respostas não eram dicotomizadas em sim ou não os entrevistados poderiam escolher mais de uma alternativa conforme o município organizava o serviço de referência e contra-referência.

Partindo do pressuposto que a Secretaria Municipal da Saúde (SMS) é responsável pelo planejamento, coordenação e avaliação das ações de saúde bucal em cada município, esta foi designada como referência para efeito de comparação das respostas e emissão de juízo de valor. Após a obtenção do percentual de resposta de cada pergunta foi possível analisar o processo de trabalho do sistema de referência e contra-referência da área da odontologia evidenciando as potencialidades e limitações da interface dos níveis de governabilidade municipal: SMS e coordenador das UBS.

O projeto foi aprovado pelo Comitê de Ética da ULBRA-CANOAS protocolo: CEP-ULBRA 2005$229 \mathrm{H}$ e antes da realização da entrevista os objetivos do projeto eram explicados aos entrevistados onde autorizavam a realização assinando o Termo de Consentimento Livre e Esclarecido. A análise estatística foi do tipo exploratória descritiva com apresentação das freqüências simples.

\section{RESULTADOS E DISCUSSÃO}

A análise do processo de trabalho do sistema de saúde permite compreender como estão organizadas as ações que impactam positivamente no desempenho das unidades de saúde e identificar limitações do serviço, permitindo o replanejamento das ações realizadas "desatando" os nós críticos que interferem para o bom atendimento à comunidade. Logo, neste estudo para construção da análise os pesquisadores preferiram realizar entrevistas com questionário prétestado a questionários autoaplicáveis intencionando obter informações de $100 \%$ da amostra selecionada para de fato compreender como um todo a articulação das ações programadas pelas SMS(s) e UBS(s), o que foi contemplado neste estudo.

Para construir e efetivar o sistema de referência e contra-referência a(s) na área da saúde, e mais especificamente na área da Odontologia, as SMS(s) devem estar articuladas com Secretaria Estadual da Saúde e com o Ministério da Saúde constituindo as interfaces do sistema nacional de saúde. ${ }^{9}$ Porém a interface avaliada no estudo foi aquela entre o gestor municipal e os gestores das unidades básicas de saúde, ou seja, o gerenciamento entre estes dois níveis de gestão em nível municipal.
É pertinente salientar que o nosso trabalho não avaliou a eficácia do sistema e sim faz a análise da existência de unidades de referência e estruturação do processo de trabalho fazendo um comparativo entre dois níveis de gestão que atuam na "ponta" do sistema de saúde público de forma normativa. ${ }^{10}$ Logo, foi possível identificar em nossos resultados quantitativos, na Tabela 1, que 41\% (5) das SMS(s) afirmaram possuir sistema de referência e contra-referência es-

Tabela 1 - Distribuição segundo a estruturação do sistema de referência e contra referência dos municípios da Coordenadoria Regional estudada (respostas múltiplas).

\begin{tabular}{|c|c|c|}
\hline Variáveis & SMS n (\%) & UBS n (\%) \\
\hline \multicolumn{3}{|c|}{$\begin{array}{l}\text { Existe sistema de referência e contra-referência } \\
\text { no município }\end{array}$} \\
\hline Sim & $05(41,67)$ & $07(58,33)$ \\
\hline Não & $07 \quad(58,33)$ & $05(41,67)$ \\
\hline Total & $12(100,00)$ & $12(100,00)$ \\
\hline \multicolumn{3}{|c|}{$\begin{array}{l}\text { Qual ou quais são as unidades de referência } \\
\text { do município }\end{array}$} \\
\hline $\begin{array}{l}\text { Centro de especialidade } \\
\text { odontológica }\end{array}$ & $00(00,00)$ & $02(06,67)$ \\
\hline Hospital & $08(42,11)$ & $09(30,00)$ \\
\hline Universidade & $04(21,05)$ & $10(33,33)$ \\
\hline Outra UBS & $01 \quad(05,26)$ & $00(00,00)$ \\
\hline Setor privado & $06(31,58)$ & $09(30,00)$ \\
\hline \multicolumn{3}{|l|}{ Total } \\
\hline \multicolumn{3}{|c|}{$\begin{array}{l}\text { Quais são as especialidades odontológicas } \\
\text { contempladas nos locais de referência }\end{array}$} \\
\hline Endodontia & $08(25,00)$ & $03(15,00)$ \\
\hline Cirurgia & $10(31,25)$ & $07(35,00)$ \\
\hline Radiologia & $07 \quad(21,88)$ & $05(25,00)$ \\
\hline Outros & $07(21,88)$ & $05(25,00)$ \\
\hline \multicolumn{3}{|c|}{$\begin{array}{l}\text { Quais são os critérios utilizados para realizar os } \\
\text { encaminhamentos }\end{array}$} \\
\hline Idade & $01(06,67)$ & $00(00,00)$ \\
\hline Estado gestacional & $01 \quad(06,67)$ & $00(00,00)$ \\
\hline Gravidade do caso & $02(13,33)$ & $01 \quad(12,50)$ \\
\hline Tipo de procedimento oferecido & $00(00,00)$ & $00(00,00)$ \\
\hline Pacientes especiais & $01 \quad(06,67)$ & $00(00,00)$ \\
\hline Parceria com outras instituições & $01 \quad(06,67)$ & $07(87,50)$ \\
\hline Não há critério & $09(60,00)$ & $00(00,00)$ \\
\hline \multicolumn{3}{|c|}{$\begin{array}{l}\text { Existe um sistema de acompanhamento pela UBS dos } \\
\text { tratamentos realizados pelo local de referência }\end{array}$} \\
\hline Sim & $11(91,67)$ & $12(100,00)$ \\
\hline Não & $01 \quad(08,33)$ & $00(00,00)$ \\
\hline
\end{tabular}

SMS - Secretaria Municipal da Saúde UBS - Unidade Básica de Saúde 
truturado na área da Odontologia onde a principal unidade utilizada para referência é o hospital (42\%), porém os coordenadores das UBS(s) responderam que em 58\% (7) dos municípios existe o sistema e a principal unidade de referência é a Universidade $(33 \%, \mathrm{n}=10)$. Verificam-se divergências nas repostas o que não poderia ocorrer, pois o processo de trabalho deve ser construído coletivamente participando todas as partes envolvidas na prestação dos serviços culminando em respostas coincidentes. ${ }^{11}$ Logo, o que pode explicar as diferentes respostas entre os dois níveis de gestão é que as UBS(s) por atuarem na linha de frente, ou seja, diretamente com os usuários do SUS e tendo a missão de proporcionar resolutividade as suas necessidades podem ter estruturado o seu sistema de referência de forma diferente daquele pactuado com SMS, pois o planejamento deste processo não deve ter sido realizado de modo coletivo e sim de forma normativa pela SMS não dando conta das reais necessidades. Outra hipótese que pode estar contribuindo para as divergências de respostas é que as UBS(s) estão apenas encaminhando os usuários com as referidas necessidades de tratamento odontológico, desconhecendo se a unidade de referência está efetivamente acolhendo e vinculando-se a este usuário. Fica evidente a necessidade de uma efetiva coordenação do serviço para um apropriado referenciamento dos pacientes com necessidades odontológicas.

A maior taxa de respostas para o referenciamento de pacientes para Universidade, dada pelas UBS(s), pode ser elucidado pela missão comunitária que as universidades possuem, sendo materializada pelos projetos de extensão, que podem estar absorvendo parte da demanda referenciada. ${ }^{12}$ No entanto, sem um efetivo planejamento para estabelecer as "trilhas" que todos os envolvidos no sistema de saúde da área da odontologia (gestores, prestadores de serviço e unidades de ensino bem como pacientes) devam seguir podem ocasionar insatisfação dos pacientes. ${ }^{13}$ Nesta lógica uma melhor interface entre o atendimento odontológico da atenção primária e secundária deve ser estabelecido. ${ }^{8,9}$

O trabalho demonstra que os hospitais, com alta taxa de resposta dada pelas SMS(s), constituem a principal unidade de referência dos municípios. Estes resultados demonstram que os hospitais ainda são os principais responsáveis pelo atendimento de média complexidade na área da Odontologia. Contudo, é necessário deixar claro que os hospitais, como unidades de referência para Odontologia, são essenciais para o atendimento à população considerando sua estrutura para procedimentos sob anestesia geral bem como aqueles casos de emergência. Atualmente muitas unidades hospitalares disponibilizam apenas atenção odontológica de Alta Complexidade e mais especificamente na área da Cirurgia e Traumatologia Bucomaxilo Facial. ${ }^{14}$ Os gestores podem expandir o atendimento odontológico nas unidades hospitalares com recursos provenientes da Política Brasil Sorridente aliando aos recursos humanos existentes nas faculdades de odontologia do Brasil. ${ }^{7,11,15}$

O privilégio de alguns municípios da Coordenadoria estudada encaminharem seus pacientes para universidades é, sem dúvida um avanço, constituindose em uma potencialidade na prestação de serviços de média complexidade da área da odontologia. As universidades podem dar uma atenção diferenciada dos hospitais por possuírem recursos humanos qualificados e materiais adequados para o atendimento odontológico nas especialidades de Endodontia, Periodontia e Atendimento de Pacientes com Necessidades Especiais. Para isso está posto o desafio de integrar o ensino odontológico com os serviços prestados pelo Sistema Único de Saúde (SUS), visto que as Diretrizes Curriculares dos Cursos de Odontologia do Brasil apontam para uma formação do Cirurgião-Dentista que deve ser integrada ao SUS. ${ }^{12}$

Outro questionamento realizado nos municípios foi a respeito do número de cotas que o centro de referência disponibiliza para cada unidade básica de saúde e quantos atendimentos são realizados mensalmente no que tange as responsabilidades de cada especialidade odontológica (prótese, cirurgia, endodontia e periodontia). Foi relatado, tanto pelas SMS e as UBS, que não existe cotas e nenhum controle é efetuado de quantos atendimentos são realizados mensalmente pelo Centro de referência daqueles encaminhamentos realizados. Os dados encontrados apontam para uma interface fragilizada, com limitações e sérias dificuldades na organização e controle da demanda o que pode levar insatisfação aos usuários e baixa resolutividade do setor de prestação de serviço de média complexidade, constituindo um nó crítico do processo de trabalho do sistema de referência e contra-referência da área da odontologia nesta coordenadoria. Nossos resultados vão ao encontro das Diretrizes da Política Nacional de Saúde Bucal, ${ }^{2}$ pois os serviços odontológicos da atenção secundária (média complexidade) não acompanharam a evolução dos serviços prestados na Atenção Básica (baixa complexidade).

Leal e Tomita ${ }^{15}$ encontraram que o principal foco 
do atendimento Odontológico no Brasil é na Atenção Básica e de forma incipiente acontece a estruturação do atendimento de Média Complexidade. Portanto, há necessidade que os municípios organizem a sua atenção básica na área odontológica para progredir de maneira satisfatória na organização e gerenciamento da média complexidade com a implementação de um Centro de Especialidades Odontológicas normatizado pelas Portarias: 1.571, 1.572 e $1.579 \mathrm{pu}-$ blicadas no Diário Oficial de 29 de Julho de 2004. 4,3,16 Talvez uma alternativa plausível para que os municípios superarem dificuldades relativas à infra-estrutura satisfatória, contratação de pessoal especializado e remuneração a altura do padrão do serviço a ser prestado, seja a efetivação de um Consórcio Intermunicipal de Saúde onde viabilizaria a incorporação de um maior número de beneficiários. ${ }^{17}$ Figueiredo \& Goes, $2009^{10}$ relataram que quanto menor o Índice de Desenvolvimento Humano do município e menor porte populacional pior é o desempenho do Centro de Especialidade Odontológica no Estado de Pernambuco - Brasil o que demonstra que a evolução da saúde bucal da população também necessita de melhores condições de renda, escolaridade e melhor expectativa de vida o que deve, também, ser considerado para a evolução do sistema de referência e contra-referência da odontologia.

Ainda no contexto da estruturação organizacional do sistema de referência e contra-referência os dois níveis de gestão foram questionadas a respeito de quais os critérios adotados para realizar os devidos encaminhamentos bem como a existência de um sistema de acompanhamento dos tratamentos realizados. As SMS(s) responderam com maior percentual $(60 \%)$ que não existem critérios para efetuarem os encaminhamentos em contra partida as UBS(s) responderam com maior prevalência de $87,5 \%$ que o principal critério seguido para efetuar os encaminhamentos foi a parceria estabelecida com outra instituição. Os dados demonstram mais uma vez a fragilidade no processo de trabalho do sistema de referência e contra-referência existentes nas SMS(s) avaliadas e, a preocupação das UBS(s) em desafogar o gargalo da média complexidade na área da Odontologia de seu território de abrangência. Com esta estrutura limitada seria interessante que as SMS(s) em conjunto com as UBS(s) definissem os critérios para priorizar os pacientes que necessitassem de atendimento na unidade de referência (UR). As Unidades de Saúde poderiam utilizar como critérios a gravidade do caso, a idade dos pacientes, estado gestacional, pacientes especiais e tipo de serviço disponibilizado pela UR (cirurgia, endodontia, prótese e pacientes com necessidades especiais). Ficando claro que estes critérios necessitam de uma avaliação respeitando as individualidades de cada micro-região. ${ }^{18}$ Contudo podem constituir uma ferramenta importante para alicerçar a estruturação do sistema de referência e contra-referência do sistema público de saúde, com declaradas evidências de fragilidades na sua organização e processo de trabalho.

Como potencialidades a nossa análise detectou que mais de $90 \%$ das SMS(s) e UBS(s) informaram que à existência de um sistema de acompanhamento dos tratamentos realizados pelas UR. A informatização dos agendamentos bem como o prontuário único e eletrônico poderiam auxiliar no controle deste sistema complexo, desta forma refinando a metodologia de avaliação e deixando para o passado as avaliações utilizando indicadores empíricos como número de pessoas que constituem a fila de espera..$^{15}$ Porém em nosso trabalho não foi avaliada a forma de acompanhamento e controle das ações não havendo a possibilidade de comparações.

Quando as CMS(s) foram questionadas a respeito de qual(is) era(am) o(s) local(is) de referência considerando as necessidades odontológicas para os encaminhamentos efetuados pelas Unidades Básicas, as respostas foram variadas conforme demonstra a Tabela 2. Mesmo aquelas que responderam anteriormente que não existia em seu município o sistema de referência e contra-referência, agora salientaram que

Tabela 2 - Relação da demanda qualitativa de procedimentos odontológicos com necessidade de encaminhamento das Unidades Básicas para os locais de referência.

\begin{tabular}{|c|c|c|c|c|c|}
\hline \multirow{2}{*}{$\begin{array}{c}\text { Local de referência } \\
\text { utilizados pelos } \\
\text { municípios }\end{array}$} & \multicolumn{4}{|c|}{$\begin{array}{c}\text { Demanda qualitativa de procedimentos odontológicos com necessidades } \\
\text { de encaminhamento das Unidades Básicas }\end{array}$} \\
\cline { 2 - 6 } & Radiografias & Endodontias & Biópsias & Extrações complexas & Outros \\
\hline Hospital & $02(13,33)$ & $00(00,00)$ & $06(40,00)$ & $03(20,00)$ & $04(26,67)$ \\
\hline Universidade & $03(11,11)$ & $06(22,22)$ & $07(25,93)$ & $06(22,22)$ & $05(18,52)$ \\
\hline Outra cidade & $04(12,90)$ & $09(29,03)$ & $08(25,81)$ & $05(16,13)$ & $05(16,13)$ \\
\hline
\end{tabular}


encaminham seus pacientes para um ou mais locais colocados como opção de resposta, demonstrando a necessidade da existência do serviço e da efetiva organização. Para os hospitais são encaminhados os procedimentos cirúrgicos como as biópsias e as extrações complexas ocorrendo em $60 \%$ (9) dos municípios. Apesar de haver respostas por parte dos respondentes, é oportuna salientar que aqueles gestores que responderam não possuir um sistema de referência e contra-referência da área da odontologia no município $(58 \%)$, responderam a entrevista com vistas a satisfazer o entrevistador, pois não havendo o sistema não existe qualquer tipo de orientação emanada pelo SMS, logo materializa a inexistência de planejamento dos municípios da coordenadoria estudada. Objetivamente a responsabilidade de planejar, estruturar e coordenar ${ }^{11}$ as UBS no que tange aos encaminhamentos dos pacientes para os atendimentos de média complexidade é da Secretaria Municipal da Saúde (Gestor) com a co-participação dos Coordenadores das UBS(s).

Também foi possível verificar que os hospitais não disponibilizam procedimentos de endodontia, periodontia, etc. No entanto, quando analisamos os dados referentes à universidade verifica-se que existe uma uniformidade de encaminhamentos das necessidades de endodontia (22\%), biópsias (25\%) e extrações complexas $(22 \%)$ demonstrados na Tabela 2. Para outras cidades o maior número de encaminhamentos ocorre na área da endodontia (29\%). Nossos dados revelam a limitação dos hospitais em prestar assistência nas outras áreas da odontologia preconizadas na Política Brasil Sorridente, ${ }^{6}$ pois a maioria das SMS(s) que participaram do estudo respondeu que as necessidades cirúrgicas do município são encaminhadas para os hospitais que mesmo com suas limitações para a diversidade de procedimentos odontológicos, dão conta da demanda reprimida da odontologia. A prestação de serviços do hospital pode ser potencializado disponibilizando atendimento odontológico para população interna e externa ao hospital, aproveitando para incluir o atendimento na área da Odontologia Hospitalar, disponibilizando a prestação de serviços como: endodontia, periodontia e atendimentos de pacientes com necessidades especiais bem como procedimentos restauradores, de promoção e prevenção da saúde bucal até mesmo em Unidades Intensivas de Tratamento (UTI).$^{19}$ Os hospitais são ferramentas importantes para a construção do sistema de referência e contra-referência em todas as áreas da saúde no município. Pela facilidade de acesso que os hospitais
Tabela 3 - Protocolo estabelecido pelo Centro de Referência após a realização do tratamento requisitado pela Unidade Básica de Saúde

\begin{tabular}{|l|c|}
\hline \multicolumn{1}{|c|}{ Variáveis } & n (\%) \\
\hline $\begin{array}{l}\text { Destino do paciente após o tratamento realizado no } \\
\text { centro de referência }\end{array}$ & $04(33,33)$ \\
\hline $\begin{array}{l}\text { São reencaminha as unidades básicas } \\
\text { Continua o tratamento no centro de } \\
\text { referência }\end{array}$ & $06(50,00)$ \\
\hline $\begin{array}{l}\text { Não há este controle } \\
\text { Protocolo seguido para a volta dos pacientes à Unidade } \\
\text { Básica de Saúde }\end{array}$ & $12(91,67)$ \\
\hline Agendamento & $02(08,33)$ \\
\hline Livre demanda
\end{tabular}

possuem nos município os gestores poderiam utilizar estas casas de saúde para a implementação da política Brasil Sorridente adequando sua estrutura física com recursos financeiros garantidos pela Coordenação Nacional de Saúde Bucal atuante em nível de Ministério da Saúde. ${ }^{7}$

Na Tabela 3 foram relatados os percentuais das respostas quando as SMS foram questionadas sobre qual é o procedimento adotado pela unidade de referência (UR) após a realização do tratamento solicitado pela UBS. Foi possível verificar que somente $33 \%$ das secretarias responderam que realizam a contrareferência e as outras $50 \%$ das SMS relataram que o paciente continua na UR até completar o tratamento e 17\% não tem controle do paciente após o final do tratamento. Ainda na Tabela 3 é possível verificar que mais de $90 \%$ dos municípios executam a contra-referência de seus pacientes através de agendamento e o restante através de livre demanda. Analisando estes aspectos fica evidente a falta de critérios para organizar este ponto de vital importância para o sistema de referência e contra-referência, sendo que uma baixo percentual de municípios (33\%) demonstrou organizar a contra-referência dos seus pacientes para efetivamente controlar e dar resolutividade aos casos recebidos na UR o que pode ser amplamente organizado através de um processo documental realizado em formulários de encaminhamento e capacitação dos recursos humanos envolvidos da rede básica e especializada. ${ }^{20}$

Lima et al. ${ }^{12}$ elaboraram critérios que podem ser utilizados para avaliação e controle do sistema de referência e contra referência: taxa de produtividade, tempo de espera para marcação de consultas e realização de procedimentos, taxa de movimentação com 
pendências (atendimento e alta), taxa de absorção das emergência pelas clínicas, percentual de prontuários devidamente preenchidos, índice de satisfação do usuário, número de contra-referência em relação aos número de referências efetuados, taxa de absenteísmo, índice de satisfação profissional e percentual de acidentes de trabalho sendo que todos os critérios permitem o replanejamento da estruturação dos serviços. Desta maneira pode ser proporcionando maior cobertura e profundidade dos serviços prestados de média complexidade. Todos estes critérios em conjunto com a análise e discussão realizado no trabalho certamente contribuirão para uma estruturação e desempenho do sistema de referência e contra-referência da área da odontologia em municípios enquadrados no sistema de gestão municipal do tipo plena ou gestão da atenção básica. ${ }^{10}$

\section{CONSIDERAÇÕES FINAIS}

Com a análise do estudo é possível afirmar que há necessidade de implantar e organizar o sistema de referência e contra-referência da área da Odontologia na Coordenadoria estudada, pois a a interface entre SMS e UBS coloca em evidência limitações do sistema. A CRS deve lançar mão de uma assessoria técnica capacitando seus recursos humanos para o planejamento e estruturação do sistema de referência e contra-referência na área da Odontologia. Também deve assumir a sua função de coordenadoria orientando um planejamento coletivo entre as SMS(s) e UBS(s) de seus respectivos municípios para criar estratégias resolutivas considerando a atual política nacional de saúde bucal - Política Brasil Sorridente. . $^{5,10,16,20}$

\section{ABSTRACT}

\section{Work process of the reference and counter- reference dentistry systems}

The aim of this study was to analyze the work process of the reference and counter-reference dentistry systems. Interviews were conducted to aid in making a comparative and normative analysis. Eight closed questions were asked, directed at the Municipal Secretaries of Health (SMS) and coordinators of Basic Health Units (UBS) from the same Regional Health Coordinator's Office (CRS), handling 12 municipalities of the state of Rio Grande do Sul. After conducting the interviews, the data were tabulated according to simple frequency. For the purpose of comparative analysis, the SMS(s) were considered as the gold standard for issuing value judgments. Once conclud- ed, the analysis identified potentials and limitations of the municipal governability interface work process. We concluded that the work process of reference and counter-reference of the dentistry system should be planned by managers from the SMS(s) and UBS(s) coordinated by the Regional Health Coordinator's Office (CRS) in order to meet the specific needs of each municipality.

\section{DESCRIPTORS}

Reference. Secondary care. Oral health.

\section{REFERÊNCIAS}

1. Ministério da Saúde. Portaria n ${ }^{\circ}$ 267, 7 de março de 2001. Define o plano de reorganização da saúde bucal na atenção básica. Diário Oficial da União 2001; 08 mar.

2. Ministério da Saúde. Diretrizes da política nacional de saúde bucal. 2004 Jan [Acessado em 201124 Maio]. Disponível em: http://189.28.128.100/dab/docs/publicacoes/geral/diretrizes_da_politica_nacional_de_saude_bucal.pdf

3. Ministério da Saúde. Portaria ${ }^{\circ}$. 1.572, de 29 de julho de 2004. Estabelece o financiamento dos Centros de Especialidades Odontológicas - CEO. Diário Oficial da União 2004; 30 jul.

4. Ministério da Saúde. Portaria n ${ }^{\circ}$. 1.571, de 29 de julho de 2004. Estabelece o pagamento de próteses dentárias totais em Laboratórios Regionais de Próteses Dentárias - LRPD. Diário Oficial da União 2004; 30 jul.

5. Pedrazzi V, Dias KRHC, Rode SM. Oral health in Brazil - Part II: Dental Specialty Centers. Braz Oral Res 2008; 22(Spec Iss 1): 18-23.

6. Pucca Junior GA, Costa JFR, Chagas LD, Silvestre RM. Oral health policies in Brazil. Braz Oral Res 2009; 23: 9-16.

7. Pucca Junior GA, Lucena EHG, Cawahisa PT. Financing national policy on oral health in Brazil in the context of the Unified Health System. Braz Oral Res 2010; 24(Spec Iss 1): 26-32.

8. Morris AJ, Burke FJ. Primary and secondary dental care: how ideal is the interface? Br Dent J 2001; 191:666-670.

9. Morris AJ, Burke FJ. Primary and secondary dental care: the nature of the interface. Br Dent J2001; 191:600-664.

10. Figueiredo N \& Goes PSA. Construção da atenção secundária em saúde bucal: um estudo sobre os Centros de Especialidades Odontológicas em Pernambuco, Brasil. Cad SaudePublica 2009; 25: 259-267.

11. Vilasbôas ALQ, Paim JS. Práticas de planejamento e implementação de políticas no âmbito municipal. Cad Saude Publica 2008; 24(6): 1239-1250.

12. Kriger L, Morita Mc. Mudanças nos cursos de Odontologia e a integração com o SUS. Rev ABENO 2004; 4: 17-21.

13. Kinnersley P, Rapport FM, Owen P, Stott N. In-house referral: a primary care alternative to immediate secondary care referral? Family Practice 1999; 16: 558-561. 
Processo de trabalho do sistema de referência e contra-referência da área da Odontologia •

Moura FRR, Bissacotti PRC, Bastos FA, Santos RB, Demarco FF, Tovo MF

14. Lima Jv, Faveret Ac, Grabois A. Planejamento participativos em organizaçoes da saúde: o caso do Hospital Geral de Bonsucesso, Rio de Janeiro, Brasil. Cad Saude Publica 2006; 22: 631-641.

15. Leal Rb, Tomita Ne. Assitência odontológica e universalização: percepção de gestores municipais. Cien Saude Colt 2006; 11: 155-160.

16. Chaves SCL, Barros SG, Cruz DN, Figueiredo ACL, Moura BLA, Cangussu MCT. Brazilian oral health policy: factors associated with comprehensiveness in health care. Rev Saude Publica 2010; 44: $1-8$.

17. Bastos FRA. O consórcio intermunicipal de saúde da região centro do RS: uma década de história. Dacasa Editora; 2005.
18. Martelli PJS, Cabral APS, Pimentel FC, Macedo CLSV, Monteiro IS, Silva SF. Análise do modelo de atenção à saúde bucal em municípios do estado de Pernambuco. Cien Saude Colet 2008; 13: 1669-1674.

19. Munro CL, Grap MJ. Oral health and care in the intensive care unit: state of science. Am J Crit Care 2004; 13: 25-34.

20. Ministério da Saúde. Manual de especialidades odontológicas em saúde bucal. Brasília: Secretaria de atenção à saúde. Departamento de Atenção Básica, 2008; 128p.

Recebido em 09/03/2010 Aceito em 15/06/2010 